\title{
Kidney Mixed Epithelial and Stromal Tumor
}

National Cancer Institute

\section{Source}

National Cancer Institute. Kidney Mixed Epithelial and Stromal Tumor. NCI Thesaurus.

Code C37263.

A rare, usually benign tumor that arises from the kidney. It belongs to the spectrum of mixed epithelial and stromal tumor family. It usually affects females. It is characterized by the presence of a biphasic pattern with tubular and cystic structures in a spindle cell stroma. Patients usually present with flank pain and hematuria. 